\title{
Comparative study on information service mode type of digital library
}

\author{
Haiyan Hao
}

(Library, Shanghai Institute of Technology, Shanghai,201418,China)

\begin{abstract}
Key words: digital library; information service; information service mode
\end{abstract}
\begin{abstract}
This paper applies mode method to carry out an in-depth study on information service modes of digital library from general to specific and from low level to high level through literal statement. The specific service mode which can best reflect and give play to information service efficiency of digital library is sought through comparative study advantages and disadvantages of different types of information service modes of digital library. Finally, the purpose of guiding Chinese digital library construction and service practice and improving information service efficiency of digital library is achieved.
\end{abstract}

\section{Foreword}

Information service mode describes elements of information service activities (information user, information server, information service content and information service strategy) and relations among these elements. Information service mode of digital library refers to combination of mutual relations among various elements of information service system of digital library (information user, information server, digital information resource, digital information platform, digital information strategy and mode). Besides, it is a working mode for information service system of digital information to carry out information service activities.

\section{Types of information service modes of digital library}

Information user, information server, information service content, information service strategy and mode are main components of information service. Their relationship degree and action mode are different. These elements and their mutual relations are main bails to distinguish different information service modes. Description of elements of information service activities and their mutual relations can form a mode. According to different division standards, information service mode of digital library shows different service mode types.

\section{Classification according to effects of information service elements}

(1) “Content-centered” service mode

It is an information service working mode oriented to information resources and centered by information service products. Information service personnel form information products through processing and adding value of information resources and provide them to information users through a strategy and mode. Currently, most libraries basically adopt such mode. Such mode highlights the status of service resource and information products, which is right. But it excessively stresses the importance of the object and neglects subjective initiative of service personnel and information users. Thus, it is to the disadvantage of improving working efficiency. As information environment of the library changes and develops, this mode seemingly lacks vitality and vigor.

\section{(2) "Librarian-centered" service mode}

It is a kind of information service working mode centered by information service personnel. Information service personnel are the core of information service personnel work. All jobs are based on the purpose of bringing benefits for information service personnel to carry out service work. Active participation ability of information users and user satisfaction are rarely considered. Users are always in the state of passive acceptance. They have no opportunities or have few opportunities to participate in selecting, processing and delivering information contents. They can only passively receive service contents provided by service personnel. Their information requirements cannot be fully reflected in the service process, so it is hard for them to gain 
information contents they really need.

(3) “User-centered" service mode

It is a kind of opportunities working mode in which information service work starts with information users and which aims to satisfy information needs of information users and solve problems. Information service work starts with information users and users are in the central status. Information knowledge products needed by users are produced in accordance with information needs of information users and information activities used to solve problems and provided to users through s strategy and mode so that users' problems can be thoroughly solved in the service activity. This mode aims to meet information needs of information users and solve problems, fully reflects subjective initiative and participation effect of information users in information service activities and overcomes shortcomings of "content-centered" mode and "librarian-centered" mode. "User-centered" service mode is the development direction and mainstream mode of information services of current and future digital libraries.

\section{Classification according to relationship between information service personnel and information users}

\section{(1) Active service mode}

Active service mode refers to the information service working mode in which service personnel in digital library analyze results, actively organize information resource, develop information products and deliver them to information users according to their tasks and purposes or users' information needs. Users can rapidly gain the information they need to reduce the time for online searching and improve information acquisition efficiency.

\section{(2) Passive service mode}

Passive service mode refers to the information service working mode in which service personnel in digital library passively organize and accumulate information resources according to users' requirements confirmed in advance and provide specific information products and services form users. It regards the library as the supreme headquarters and requires readers must gain services in the library, but readers will suffer the time limit. Thus, such mode is a passive service mode and is constrained by time and space. It seriously constrains ideology of the personnel and forms stubborn passive service concept. Under such mode, the satisfaction rate and service effects of information services can be imagined.

\section{(3) Interactive service mode}

Interactive service mode refers to a working mode in which service personnel and users jointly participate in the whole process of producing, delivering and utilizing information products and complete information service objective through interactions and communications. In this mode, the service personnel in digital library analyze results, actively organize information resources, develop information products and deliver them to information users according to their tasks and purpose or users' information needs. Information users also actively express their needs to the information organ and participate in information organization, product development and service activities of service personnel. Such interactive mode contributes to giving play to knowledge and specialty advantages of librarians and users and realizing the best service benefit.

\section{(4) Self-service mode}

Self-service service mode is a self-participation information service mode in which information users apply the computer and network to self-utilize all kinds of resources in the library to collect, identify, refine, deliver and utilize literature information to satisfy their information need and solve problems with the help of digital library and library information service personnel. Users' all information utilization activities and problem solutions are autonomously carried out on intelligent digital library information service system platform. Its advantage is to highlight users' subject status. If users' initiative is strong and their participation degree is high and service strategy and service contents have strong pertinence, these are beneficial to developing and utilizing library resources and achieving resource sharing. Service personnel mainly construct and maintain information resource management and service platform of digital library and do not directly participate in users' information activities and process. They just provide the tool, strategy and method for users to solve 
problems and guide users' information activities.

\section{Classification from technical perspective of information service}

\section{(1) Distributed service mode}

Distributed service mode utilizes internet protocol, common gateway protocol, computer collaborative technology and other modern communication technological means to support work in distributed network information environment, and regards regional digital library or digital information resource center as resource collection and service unit. Each digital library has its own retrieval system, knowledge warehouse and data warehouse. The nodes of each digital library perceive node information of other members through a common cooperation mode. Internet is regarded as the bridge among digital libraries. If network bandwidth is allowed, information real-time interaction can be realized among digital libraries, among librarians, between users and librarians, between users and experts and among users. The advantage of distributed service mode is to fully consider information resources distribution features in network environment and needs of information users, start with mutual operations and integration of diversified digital information resources, break through serious separation of original departments, comprehensively support retrieval and acquisition of distributed heterogeneous information and stress normative utilization and management of information resources so as to give full play to advantages of digital library in distributed digital information resource environment and provide comprehensive multi-level and multi-angle personalized information products and services. Distributed service mode distributes resource construction and services in multiple digital libraries, which can fully utilize library resources of each library. Since document delivery request is directly sent to the service library, information interaction is direct and convenient. Meanwhile, wide development space is provided for each library. It is more beneficial to realizing co-construction and sharing of digital resource system. However, distribution mode also gives rise to some problems such as information transfer difficulty, internet balance and user management.

\section{(2) Integrated service mode}

Information integration service mode comprehensively utilizes modern information technology on the basis of information resource integration to integrate library information resource, technological resource and human resource so as to provide users with comprehensive integration. It is a leap of distributed service mode, integrates digital information resource, technology, personnel, organizations and service functions with difference, distribution and management autonomy and achieves effective control of dispersive digital library resource system so that users can gain dynamic, problem-oriented, all-round, multi-level and diversified information service with space and time consistency to construct user-oriented efficient and comprehensive information integration service system of digital library. It aims at integration of digital library contents and products, regards functional integration as the structure, takes platform integration as technical foundation and deems human integration as fundamental guarantee to finally form uniform fundamental guarantee, one-time user authentication, seamless connection among different systems and complete integrated comprehensive platform system of digital library information service. Integration service advantages are user-oriented and task-oriented and have explicit objective or theme. It can save users' time, provide fast-paced services for users and boost information service efficiency and service level.

\section{Classification from the perspective of digital library system}

\section{(1) Distributed service mode}

Distributed service mode is an isolated service mode. Under such service mode, digital library generally develops in a relatively closed environment. Its resource construction, system construction, information product and service supply appears through independent systems. There is the lack of necessary and effective connection and coordination among various digital libraries. Various resources and service systems disperse at different surfaces and form dispersive point-to-point or point-to-surface service state. No effective connection forms among each point and surface. No effective digital library system forms. The service coverage is narrow; the opening degree is low; the service efficiency is low. 


\section{(2) Centralized service mode}

Concentrated service mode means a tangible or virtual state-level digital library or digital information resource center provides digital literature guarantee service, and users or digital library member units directly put forward information demand request. The center provides the information to the applicant after it gains the information. The users and affairs related to information supply and delivery are intensively managed by the center. Concentrated service mode requires digital library is planned and operated according to standardized requirements from a series of problems such as resource construction, system construction and transmission protocol. It has high requirements for constructors. So, it is hard to implement it. Besides, strong capital is required. But, concentrated service mode can effectively concentrate national fund input, reduce repeated construction of resources to the largest extent and greatly improve information resource utilization ratio. Due to stable financial support by the government, limited fund of digital library member units can be relieved. Meanwhile, concentrated mode is convenient for coordinated management and improves digital literature transmission rate.

\section{(3) Alliance service mode}

Digital library alliance (collaborative type) service mode means various digital libraries are organized to realize resource sharing and mutual benefit. They are based on several digital libraries and unite relevant information resource systems to establish digital library combo service mode according to commonly approved protocol and contract. In alliance service mode, various digital library members cooperate for services through certain information transfer structure according to uniform technical standards and working procedure so as to reach the purpose of resource sharing, mutual-use of service and mutual benefit. Such service mode generally forms in voluntary and spontaneous. It can be concrete organization entity or virtual organization. Digital library alliance service mainly fully shares resources and cooperates for development in such aspects as joint purchasing, joint catalogue, inter-library lending, common retrieval, resource navigation, cooperation consultation and joint training. Alliance service mode is an inexorable trend of digital library development. Digital library can continue to survive only on the basis of alliance.

\section{Development prospect of digital library information service mode}

By synthesizing features of library information service mode, it can be seen that the innovation spirit and principal line of library information service mode lie in transformation from resource-oriented information service to user-oriented information service and also lie in establishment of user-oriented information service. Currently, "user-entered" service mode is the development tendency of library information service. However, as information technology develops, service mode will change and more comprehensive mode will appear. So, information service mode innovation will continuously develop, find out new implementation point, promote and perfect development of digital library as long as information service mode innovation is based on user-centered and user-oriented principle and complies with user need.

\section{References:}

[1] Guo Haiming, Study on information service mode of digital library [J]. Information Science, 2005 (10) 1546-1551

[2] Chen Jianlong, Study on information service mode [J]. Journal of Peking University (Philosophy and Social Science), 2003 (3) 124-132

[7] Li Hong, Study on user-oriented digital library information service mode. Journal of Information, 2007 (8) 134-13

[9] Zhang Xiaolin, Distributed digital library mechanism [J]. Information Science, 2002(1)63-70

[10] Qian Zhiyong, Cooperative digital reference consultation service of American library [J] Library, 2003(5)39-4

[12] Huo ZHongwen, Zhang Jie, Information integration service development strategy [J]. Information Studies: Theory \& Application, 2001 (l) 1-5 